Finally if $\lambda$ is such a sequence that

$$
\lambda_{n}=2 \cdot 3^{-1} \cdot 3^{-n} \quad \text { whenever } n \in \omega
$$

then subsum $\lambda$ is the Cantor ternary set of Lebesgue measure zero.

University of California, Berkeley

\title{
BOUNDS FOR THE MODULI OF THE ZEROS OF A POLYNOMIAL
}

FULTON KOEHLER

1. Introduction. Let $f(z)=a_{0}+a_{1} z+\cdots+a_{n} z^{n}, a_{0} \neq 0, a_{n} \neq 0$. Following the notation of Ostrowski [1], we define the Newton diagram of $f(z)$ as the broken line $D$ in the $(x, y)$-plane with the following properties:

(1) $D$ extends from the point $x=0, y=-\log \left|a_{0}\right|$ to the point $x=n, y=-\log \left|a_{n}\right|$;

(2) $y_{\nu} \leqq\left(y_{\nu-1}+y_{\nu+1}\right) / 2(\nu=1,2, \cdots, n-1)$, where $y_{\nu}$ is the ordinate of $D$ for $x=\nu$;

(3) The points $\left(\nu,-\log \left|a_{\nu}\right|\right)(\nu=0,1, \cdots, n)$ all lie on or above $D$;

(4) If $\left(\nu, y_{\nu}\right)$ is a corner point of $D$, i.e., $y_{\nu}<\left(y_{\nu-1}+y_{\nu+1}\right) / 2$, then $y_{\nu}=-\log \left|a_{\nu}\right|$.

Values of $\nu$ for which $\left(\nu,-\log \left|a_{\nu}\right|\right)$ is a corner point or an end point of $D$ are called principal indices of $f(z)$. Let $y_{\nu}=-\log T_{\nu}$ so $T_{\nu}=\left|a_{\nu}\right|$ when $\nu$ is a principal index, and let $R_{\nu}=T_{\nu-1} / T_{\nu}$. The slope of $D$ in the interval $(\nu-1, \nu)$ is $\log R_{\nu}$.

Let the zeros of $f(z)$ be denoted by $z_{1}, z_{2}, \cdots, z_{n}$ with $0<\left|z_{1}\right|$ $\leqq\left|z_{2}\right| \leqq \cdots \leqq\left|z_{n}\right|$, and let $b_{\boldsymbol{k}}^{n}$ and $B_{\boldsymbol{k}}^{n}(k=1,2, \cdots, n)$ be the minimum and maximum respectively of $\left|z_{k}\right| / R_{k}$ for all polynomials of degree $n$ which do not vanish at the origin. Ostrowski [1] has shown, by considering the polynomial $z^{n} f(1 / z)$, that

$$
B_{k}^{n}=1 / b_{n-k+1}^{n} \text {. }
$$

Ostrowski proved that $b_{1}^{n}$ is the positive root of the equation $1=x$ $+x^{2}+\cdots+x^{n}$, that $b_{n}^{n}$ is equal to $1 / n$, and that

Presented to the Society, April 24, 1953; received by the editors March 10, 1953 and, in revised form, September 28, 1953. 


$$
\left|z_{k}\right| / R_{k}>1-(1 / 2)^{1 / k} \quad(k=2,3, \cdots, n-1) .
$$

He proved this last inequality for the zeros of a Taylor's series, with $>$ replaced by $\geqq$, and for all values of $k$, and showed that in this case the bound is the best possible. He also proved that $b_{2}^{3}=1 / 3$.

The main result to be proved in this paper is that $b_{k}^{n}$ is equal to the positive root of the equation

$$
1=\left[\begin{array}{l}
k \\
1
\end{array}\right] x+\left[\begin{array}{l}
k \\
2
\end{array}\right] x^{2}+\cdots+\left[\begin{array}{c}
k \\
n-k+1
\end{array}\right] x^{n-k+1}=\phi_{k}^{n}(x),
$$

where

$$
\left[\begin{array}{l}
k \\
p
\end{array}\right]=\left(\begin{array}{c}
k+p-1 \\
p
\end{array}\right) .
$$

It may be noted that $\phi_{k}^{n}$ is a partial sum of the series for $(1-x)^{-k}-1$, and that the equation $(1-x)^{-k}-1=1$ has as its only positive root the value $x=1-(1 / 2)^{1 / k}$ which appears in (2).

A problem which will be seen to be related to this result is the following, considered by Van Vleck [2]:1 given $a_{0}, a_{1}, \cdots, a_{k-1}$, $a_{h} \neq 0(k \leqq h \leqq n)$, to prove the existence of an upper bound for $\left|z_{k}\right|$. Van Vleck proves the existence of such an upper bound for any $h$, and he shows that, when $h=k$, such a bound will be the positive root $\rho$ of the equation ${ }^{2}$

$$
\left|a_{k}\right| \rho^{k}=\sum_{\lambda=0}^{k-1}\left(\begin{array}{l}
n-\lambda \\
k-\lambda
\end{array}\right)\left|a_{\lambda}\right| \rho^{\lambda} .
$$

In this paper we give a similar explicit determination of such a bound for any $h$.

We state here two lemmas which are proved in Ostrowski's paper.

LEMMA 1. If $k$ and $l$ are two consecutive principal indices $(k<l)$, then

$$
\begin{gathered}
\left|\frac{a_{k}}{a_{l}}\right|=R_{l}^{l-k}, \\
\left|\frac{a_{\nu}}{a_{k}}\right| \leqq R_{l}^{k-\nu}, \quad\left|\frac{a_{l}}{a_{\nu}}\right| \geqq R_{l}^{\nu-l} \quad(k \leqq \nu \leqq l), \\
\left|\frac{a_{\nu}}{a_{l}}\right|<R_{l}^{l-\nu}, \quad\left|\frac{a_{\nu}}{a_{k}}\right|<R_{l}^{k-\nu} \quad(\nu<k, \nu>l) .
\end{gathered}
$$

1 The author wishes to thank the referee for calling to his attention the paper of Van Vleck.

2 See also [3, p. 110 and p. 115]. 
LEMma 2. By a transformation of the form $g(z)=a f(b z)$, which leaves the ratios $\left|z_{k}\right| / R_{k}$ unchanged, the segment of the Newton diagram for $g(z)$ in any unit interval $(\nu-1, \nu)$ can be made to fall on the $x$ axis.

2. Let homogeneous polynomials $S_{\nu}\left(z_{1}, z_{2}, \cdots, z_{m}\right)$ and $H_{\nu}\left(z_{1}, z_{2}\right.$, $\left.\cdots, z_{m}\right)$ be defined by the formulas:

$$
\begin{aligned}
\left(1-z_{1} t\right)\left(1-z_{2} t\right) \cdots\left(1-z_{m} t\right) & =\sum_{\nu=0}^{\infty}(-1)^{\nu} S_{\nu} t^{\nu}, \\
\left(1-z_{1} t\right)^{-1}\left(1-z_{2} t\right)^{-1} \cdots\left(1-z_{m} t\right)^{-1} & =\sum_{\nu=0}^{\infty} H_{\nu} t^{\nu} .
\end{aligned}
$$

Multiplication of corresponding members in these two formulas gives

$$
H_{\nu}-S_{1} H_{\nu-1}+S_{2} H_{\nu-2}-\cdots \pm S_{\nu}=0 \quad(\nu=1,2,3, \cdots) .
$$

Let us consider the polynomial

$$
K_{\nu \alpha}=H_{\nu}-S_{1} H_{\nu-1}+\cdots+(-1)^{\alpha} S_{\alpha} H_{\nu-\alpha} \quad(\nu, \alpha=0,1,2, \cdots) .
$$

This is a homogeneous polynomial in $z_{1}, z_{2}, \cdots, z_{m}$ of degree $\nu$ and is therefore the sum of terms of the form $z_{1}^{\beta_{1}} z_{2}^{\beta_{2}} \cdots z_{m}^{\beta_{m}}$, where $\beta_{1}, \beta_{2}, \cdots, \beta_{m}$ are non-negative integers whose sum is $\nu$. Let $\lambda$ be the number of the $\beta$ 's which are positive. Then the coefficient of $z_{1}^{\beta_{1}} z_{2}^{\beta_{2}} \cdots z_{m}^{\beta_{m}}$ in $K_{\nu \alpha}$ is equal to

$$
1-\left(\begin{array}{l}
\lambda \\
1
\end{array}\right)+\left(\begin{array}{l}
\lambda \\
2
\end{array}\right)-\cdots+(-1)^{\alpha}\left(\begin{array}{l}
\lambda \\
\alpha
\end{array}\right)
$$

where it is understood that

$$
\left(\begin{array}{l}
\lambda \\
p
\end{array}\right)=0
$$

It therefore follows that, if similar terms in $K_{\nu \alpha}$ are combined, all coefficients are positive if $\alpha$ is even and negative if $\alpha$ is odd. Since

$$
K_{\nu \alpha}=K_{\nu, \alpha-1}+(-1)^{\alpha} S_{\alpha} H_{\nu-\alpha},
$$

the absolute value of the coefficient of any term in $K_{\nu \alpha}$ is not greater than the coefficient of that term in $S_{\alpha} H_{\nu-\alpha}$, i.e., $K_{\nu \alpha}$ is dominated by $S_{\alpha} H_{\nu-\alpha}$. Hence,

$$
\begin{aligned}
\left|K_{\nu \alpha}\right|=\left|\sum_{\sigma=0}^{\alpha}(-1)^{\sigma} S_{\sigma} H_{\nu-\sigma}\right| \leqq\left(\begin{array}{c}
m \\
\alpha
\end{array}\right)\left[\begin{array}{c}
m \\
\nu-\alpha
\end{array}\right] M^{\nu} \\
\\
(\nu, \alpha=0,1,2, \cdots),
\end{aligned}
$$


where

$$
M=\max \left\{\left|z_{1}\right|,\left|z_{2}\right|, \cdots,\left|z_{m}\right|\right\} .
$$

3. Let $f(z)=\sum_{\nu=0}^{n} a_{\nu} z^{\nu}$ have zeros $z_{1}, z_{2}, \cdots, z_{n}$ with $\left|z_{1}\right| \leqq\left|z_{2}\right|$ $\leqq \cdots \leqq\left|z_{n}\right|$, let $\rho_{\nu}=1 / z_{\nu}$, and let $f(z)$ be written in the form

$$
\begin{aligned}
f(z) & =\sum_{\nu=0}^{n} a_{\nu} z^{\nu} \\
& =\left(\gamma_{0}+\gamma_{1} z+\cdots+\gamma_{k-1} z^{k-1}\right)\left(1-\rho_{k} z\right)\left(1-\rho_{k+1} z\right) \cdots\left(1-\rho_{n} z\right) .
\end{aligned}
$$

By equating coefficients in the equations

$$
\begin{aligned}
& a_{0}+a_{1} z+\cdots+a_{n} z^{n} \\
& =\left(\gamma_{0}+\gamma_{1} z+\cdots+\gamma_{k-1} z^{k-1}\right)\left(1-S_{1} z+S_{2} z^{2}-\cdots\right), \\
& \gamma_{0}+\gamma_{1} z+\cdots+\gamma_{k-1} z^{k-1}
\end{aligned}
$$

$$
=\left(a_{0}+a_{1} z+\cdots+a_{n} z^{n}\right)\left(1+H_{1} z+H_{2} z^{2}+\cdots\right),
$$

where $S_{i}=S_{i}\left(\rho_{k}, \rho_{k+1}, \cdots, \rho_{n}\right)$ and $H_{i}=H_{i}\left(\rho_{k}, \rho_{k+1}, \cdots, \rho_{n}\right)$ are defined as in (4), we obtain

$$
a_{\nu}=\sum_{\sigma=0}^{\nu}(-1)^{\sigma} S_{\sigma} \gamma_{\nu-\sigma} \quad(\nu=0,1,2, \cdots, n)
$$

and

$$
\gamma_{\nu}=\sum_{\sigma=0}^{\nu} H_{\sigma} a_{\nu-\sigma} \quad(\nu=0,1,2, \cdots, n),
$$

letting $\gamma_{\nu}=0$ for $\nu \geqq k$.

For $\nu \geqq k$ we can obtain from (8) and (9) an expression for $a_{\nu}$ in terms of $a_{0}, a_{1}, \cdots, a_{k-1}$ as follows:

$$
\begin{aligned}
a_{\nu} & =\sum_{\sigma=\nu-k+1}^{\nu}(-1)^{\sigma} S_{\sigma} \gamma_{\nu-\sigma}=\sum_{\sigma=\nu-k+1}^{\nu} \sum_{\rho=0}^{\nu-\sigma}(-1)^{\sigma} S_{\sigma} H_{\rho} a_{\nu-\sigma-\rho} \\
& =\sum_{\lambda=0}^{k-1} \sum_{\sigma=\nu-k+1}^{\nu-\lambda}(-1)^{\sigma} S_{\sigma} H_{\nu-\lambda-\sigma} a_{\lambda} \\
& =-\sum_{\lambda=0}^{k-1} \sum_{\sigma=0}^{\nu-k}(-1)^{\sigma} S_{\sigma} H_{\nu-\lambda-\sigma} a_{\lambda},
\end{aligned}
$$

the last step being justified by (5). Hence, by (6),

$$
\left|a_{\nu}\right| \leqq \sum_{\lambda=0}^{k-1}\left(\begin{array}{c}
n-k+1 \\
\nu-k
\end{array}\right)\left[\begin{array}{c}
n-k+1 \\
k-\lambda
\end{array}\right] \rho^{\nu-\lambda}\left|a_{\lambda}\right|
$$


where $\rho=\left|\rho_{k}\right|$.

The above equation (10) and inequality (11) are given by Van Vleck $[2$, p. $115,(16)]$ for the case $\nu=k$. He also gives an equation $[2$, p. $115,(17)]$ which is an implicit form of our equation (10). By the use of (11) we can now give an explicit solution for the general problem considered by Van Vleck which was mentioned earlier. Namely, if $a_{0}, a_{1}, \cdots, a_{k-1}, a_{\nu} \neq 0(k \leqq \nu \leqq n)$ are given, then $\left|z_{k}\right|^{-1}$ is at least equal to the positive root $\rho$ of the equation

$$
\left|a_{\nu}\right|=\left(\begin{array}{c}
n-k+1 \\
\nu-k
\end{array}\right) \sum_{\lambda=0}^{k-1}\left[\begin{array}{c}
n-k+1 \\
k-\lambda
\end{array}\right]\left|a_{\lambda}\right| \rho^{\nu-\lambda} .
$$

We now consider the problem of determining $b_{\boldsymbol{k}}^{n}$ and $B_{\boldsymbol{k}}^{n}$. Let $n$ be considered fixed and let $\phi_{k}(x)=\phi_{k}^{n}(x)$ be defined by (3) and let $r_{k}$ be the positive root of the equation $\phi_{k}(x)=1$. Since $\phi_{k}(x)$ is monotonically increasing for $x>0$, the conditions $x>0, \phi_{k}(x) \geqq 1$ imply that $x \geqq r_{k}$.

By Lemma 2, we may assume without loss of generality that the Newton diagram for $f(z)$ lies along the $x$-axis in the interval $(k-1, k)$. Then $R_{k}=1,\left|a_{\nu}\right|=1$ for some $\nu \geqq k$ and, by Lemma $1,\left|a_{h}\right| \leqq 1$ for all $h$. With this value of $\nu,(11)$ gives

$$
\begin{aligned}
1 & \leqq \sum_{\lambda=0}^{k-1}\left(\begin{array}{c}
n-k+1 \\
\nu-k
\end{array}\right) \rho^{\nu-k}\left[\begin{array}{c}
n-k+1 \\
k-\lambda
\end{array}\right] \rho^{k-\lambda} \\
& =\left(\begin{array}{c}
n-k+1 \\
\nu-k
\end{array}\right) \rho^{\nu-k} \phi_{n-k+1}(\rho) .
\end{aligned}
$$

It is clear from the definition that $r_{k} \leqq 1 / k$ since the first term in $\phi_{k}(x)$ is $k x$. If $\rho<r_{n-k+1} \leqq(n-k+1)^{-1}$, then

$$
\left(\begin{array}{c}
n-k+1 \\
\nu-k
\end{array}\right) \rho^{\nu-k} \leqq 1,
$$

$\phi_{n-k+1}(\rho)<1$, and there is a contradiction of (12). Hence $\rho \geqq r_{n-k+1}$, $\left|z_{k}\right| \leqq r_{n-k+1}^{-1}$.

In order to show that $\left|z_{k}\right| / R_{k}$ can actually equal $r_{n-k+1}^{-1}$, we define $f(z)$ by $(7)$ with $\rho_{k}=\rho_{k+1}=\cdots=\rho_{n}=r_{n-k+1}, a_{0}=a_{1}=\cdots=a_{k-1}=1$. We then have, from (9),

$$
\begin{aligned}
a_{k} & =-\sum_{\sigma=1}^{k} H_{\sigma} a_{k-\sigma}=-\sum_{\sigma=1}^{k}\left[\begin{array}{c}
n-k+1 \\
\sigma
\end{array}\right] r_{n-k+1}^{\sigma} \\
& =-\phi_{n-k+1}\left(r_{n-k+1}\right)=-1,
\end{aligned}
$$

and, from (11), for $\nu>k$, 


$$
\left|a_{\nu}\right| \leqq\left(\begin{array}{c}
n-k+1 \\
\nu-k
\end{array}\right) r_{n-k+1}^{\nu-k} \phi_{n-k+1}\left(r_{n-k+1}\right)<1 .
$$

Hence $R_{k}=1$. Since $f(z)$ has $n-k+1$ zeros equal to $r_{n-k+1}^{-1}$, it can have at most $k-1$ zeros less than $r_{n-k+1}^{-1}$. Therefore, $\left|z_{k}\right| \geqq r_{n-k+1}^{-1}$ and, from what has been proved before, the equality sign must hold. It follows that

$$
B_{k}^{n}=r_{n-k+1}^{-1}
$$

and, from (1), that

$$
b_{k}^{n}=r_{k} \quad(k=1,2, \cdots, n) .
$$

\section{REFERENCES}

1. Alexandre Ostrowski, Recherches sur la méthode de Graeffe et les zéros des polynomes et des séries de Laurent, Acta Math. vol. 72 (1940) pp. 99-257.

2. Edward B. Van Vleck, On limits to the absolute values of the roots of a polynomial, Bull. Soc. Math. France vol. 53 (1925) pp. 105-125.

3. Morris Marden, The geometry of the zeros of a polynomial in a complex variable, Mathematical Surveys, vol. 3, New York, American Mathematical Society, 1949.

University of Minnesota 